\title{
Role of Zinc metalloprotease (ZMPSTE24) on Porcine Reproductive and Respiratory Syndrome Virus (PRRSV) replication in vitro
}

Xiuqing Wang ( $\square$ xiuqing.wang@sdstate.edu )

South Dakota State University https://orcid.org/0000-0003-0491-4937

Pratik Katwal

South Dakota State University

Eric Nelson

South Dakota State University

Michael Hildreth

South Dakota State University

Shitao Li

Tulane University School of Medicine

\section{Research Article}

Keywords: virus restriction factors, ZMPSTE24, Porcine Reproductive and Respiratory Syndrome Virus (PRRSV)

Posted Date: February 21st, 2022

DOI: https://doi.org/10.21203/rs.3.rs-1322176/v1

License: (c) (1) This work is licensed under a Creative Commons Attribution 4.0 International License.

Read Full License 
1 Role of Zinc metalloprotease (ZMPSTE24) on Porcine Reproductive and

2 Respiratory Syndrome Virus (PRRSV) replication in vitro

3 Pratik Katwal ${ }^{1}$, Eric Nelson ${ }^{2}$, Michael Hildreth ${ }^{1}$, Shitao Li $^{3}$, Xiuqing Wang ${ }^{1 *}$

$4{ }^{1}$ Department of Biology and Microbiology, South Dakota State University,

5 Brookings, SD, 57007, USA

$6 \quad{ }^{2}$ Department of Veterinary and Biomedical Sciences, South Dakota State

7 University, Brookings, SD, 57007, USA

$8{ }^{3}$ Department of Microbiology and Immunology, Tulane University, New Orleans,

9 LA 70112, USA

$10 *$ Correspondence: Xiuqing.Wang@sdstate.edu 
30 Abstract

31 The transmembrane zinc metalloprotease, ZMPSTE24, works in cooperation with

32 interferon induced transmembrane protein 3 (IFITM3) to restrict entry of several

33 enveloped viruses. We investigated the role of ZMPSTE24 in porcine

34 reproductive and respiratory syndrome virus (PRRSV) replication. ZMPSTE24

35 overexpression significantly reduced PRRSV replication in MARC-145 cells.

36 Interestingly, knockdown of endogenous ZMPSTE24 did not significantly impact

37 virus replication. There was no significant difference in the percentage of PRRSV

38 positive cells and viral RNA copies at 3 hours post infection (hpi) between cells

39 transfected with ZMPSTE24-FLAG and vector control. Our results suggest that

40 ZMPSTE24 overexpression may restrict PRRSV replication at a post-entry step.

41

42 Key words: virus restriction factors, ZMPSTE24, Porcine Reproductive and

43 Respiratory Syndrome Virus (PRRSV)

44

45

46

47

48

49

50

51

52

53

54

55

56

57

58 
60 viruses at various stages of their life cycle [14]. Many enveloped viruses enter

61 host cells by receptor mediated endocytosis. Triggered by acidic $\mathrm{pH}$ within

62 endosomes, the viral fusion peptide undergoes conformational change and

63 facilitates virus fusion with endosome membrane and uncoating. Several $\mathrm{pH}$

64 sensitive viruses that depend on low $\mathrm{pH}$ for fusion have been shown to be strongly

65 inhibited by IFITM3 [15]. IFITM3 is localized in the early or late endosomes and

66 impedes virus-host membrane fusion, thereby blocking release of the genome [1,

67 3]. The restriction factor ZMPSTE24 works in cooperation with the IFITM3

68 protein to restrict entry of several enveloped viruses [5, 10, 16]. ZMPSTE24 acts

69 as a downstream effector of IFITM3 $[5,10]$. The ZMPSTE24 protein is

70 constitutively expressed and localized to inner nuclear membrane [2]. It is

71 important in the processing of precursor prelamin A into lamin A in the nuclear

72 lamina [2]. Defect in ZMPSTE24 has been shown to enhance accumulation of

73 prelamin A [4]. Currently, there are very limited studies exploring the mechanism

74 by which it restricts virus entry including its interaction with the IFITM proteins

75 and IFITM independent virus restriction.

PRRSV is an enveloped, positive sense, single stranded RNA virus

77 belonging to the family Arteriviridae within the order Nidovirales [12]. PRRSV

78 causes severe respiratory disease in young pigs and reproductive failure in sows

79 [12]. Upon attachment and internalization, PRRSV enters into the early endosome

80 in clathrin-coated vesicles and virus-endosome membrane fusion leads to virus

81 uncoating $[17,19]$. The role of IFITM3 in restricting PRRSV replication has been

82 recently reported [18]. However, very little is known whether ZMPSTE24 also

83 restricts PRRSV replication. In this present study, we investigated the role of 
ZMPSTE24 in PRRSV replication and examined whether ZMPSTE24 restricts PRRSV entry into cells.

To investigate the effect of ZMPSTE24 on PRRSV replication, we first employed the ZMPSTE24 overexpression system. ZMPSTE24 overexpression has been shown to significantly reduced influenza A virus (IAV) infection in A549 cells [5]. Briefly, MARC-145 cells cultured in a 12-well plate were transfected with $1.25 \mu \mathrm{g}$ per well of either pCMV-3Tag-8 control vector or FLAG-tagged ZMPSTE24 cloned into pCMV-3Tag-8 (ZMPSTE24-FLAG) plasmid [5] using Lipofectamine 3000 Transfection Kit (Invitrogen). Cells were incubated in complete medium (DMEM with 10\% FBS and 1\% penicillin-streptomycin) for 72 h. Then, PRRSV 23983 was added to each well at an MOI of 1. Cells and supernatant were harvested $24 \mathrm{~h}$ later. Western blot analysis was done using the following primary antibodies: anti-Flag mouse monoclonal $1^{\circ} \mathrm{Ab}$ against flag tagged ZMPSTE24 (9A3) (Cell Signaling Technology, cat. no. 8146S) at 1:1000 dilution, anti-PRRSV N monoclonal Ab SR-30 at 1:300 dilution, and mouse monoclonal anti-beta actin Ab (Sigma-Aldrich, catalog\# A2228) at 1:5000 dilution. The membrane was then incubated with Goat anti-Mouse IRDye ${ }^{\circledR}$ conjugated $2^{\circ} \mathrm{Ab}$ (LI-COR, catalog\# 926-32210) diluted to 1:5000 and observed using LI-COR Odyssey Infrared Imaging System. As shown in Fig.1A, ZMPSTE24 protein was detected in cells transfected with pCMV-3Tag-8 plasmid expressing ZMPSTE24-FLAG but not in vector control pCMV-3Tag-8 transfected cells. A lower level of expression of PRRSV N protein was detected in ZMPSTE24 overexpressing cells as compared to the vector control cells (Fig.

1A). Fifty-percent tissue culture infectious dose $\left(\mathrm{TCID}_{50}\right)$ assay was performed to determine the infectious PRRSV 23983 titer in the supernatant [9]. An average of 146-fold decrease (2.16 log reduction) in virus titer in ZMPSTE24 expressing 
110 cells was observed (Fig. 1B). To further confirm the antiviral activity of

111 ZMPSTE24, MARC-145 cells were transfected with $0.6 \mu \mathrm{g}$ of each plasmid in a

112 24-well plate and infected with PRRSV 23983 for $24 \mathrm{~h}$, as described earlier. All

113 wells were fixed with $80 \%$ acetone and incubated with 1:80 diluted FITC

114 conjugated anti-PRRSV Ab specific to nucleocapsid (N) protein (SDOW-17) at

$11537^{\circ} \mathrm{C}$ for $1 \mathrm{~h}$. The images were captured at 10x magnification using an Olympus

116 IX70 Inverted-Microscope (Epi-fluorescence and bright field).

117 Immunofluorescence staining showed fewer virus positive staining (green) in

118 ZMPSTE24 overexpressing cells as compared to the vector control transfected

119 cells (Fig. 1C). To quantify the relative abundance of PRRSV infection, cells were

120 harvested and analyzed further by flow cytometry. A significant reduction in

121 mean fluorescence intensity $(\mathrm{p}<0.05)$ was observed in ZMPSTE24

122 overexpressing cells as compared to the vector control cells at 24 hpi (Fig. 1D).

123 To test if cell viability played a role in ZMPSTE24 mediated virus restriction,

124 cytotoxicity assay was performed. MARC-145 cells cultured in a 96-well plate

125 were transfected with $0.3 \mu \mathrm{g}$ of either the vector control pCMV-3Tag- 8 or

126 ZMPSTE24-FLAG for $72 \mathrm{~h}$. Each well was treated with $10 \mu \mathrm{l}$ of CCK-8 solution

127 (Sigma, cat. no. 96992), then incubated at $37^{\circ} \mathrm{C}$ for $3 \mathrm{~h}$, and absorbance was

128 measured at $450 \mathrm{~nm}$ using a Synergy 2 plate reader (Bio-Tek). No significant

129 difference $(\mathrm{p}>0.05)$ in cell viability between vector control and ZMPSTE24

130 transfected cells was observed (Fig. 1E). Overall, the results suggest that

131 overexpression of ZMPTSE24 reduces PRRSV replication.

To further confirm the role of ZMPSTE24 in restricting PRRSV

133 replication, we employed silencing RNA mediated knockdown of endogenous

134 ZMPSTE24 approach. Briefly, MARC-145 cells cultured in a 12-well plate were

135 transfected with either negative control siRNA (Ambion, cat. no. AM4642) or 
137 concentration of 2 pmoles/ $\mu 1$ for $72 \mathrm{~h}$ using Lipofectamine RNAi max reagent

138 (Invitrogen). The cells in each well were then infected with PRRSV 23983 at an

139 MOI of 1 for $24 \mathrm{~h}$. Total RNA was extracted from cells and qRT-PCR was

140 performed to validate the ZMSPTE24 gene knockdown efficiency and to quantify

141 PRRSV gene transcript. Primer sequences specific to beta-actin, PRRSV N, and

142 ZMPSTE24 genes used in the study are available upon request. Mean fold change

143 in gene expression was calculated using the $\Delta \Delta \mathrm{CT}$ method [11]. Beta-actin was

144 used as a reference gene for comparing the fold change between two treatment

145 groups [11]. As shown in Fig. 2A, an average of 74\% knockdown of endogenous

146 ZMPSTE24 gene was achieved. An average of 1.2-fold increase in PRRSV N

147 gene transcript relative to the silencing control was observed (Fig. 2B).

148 Surprisingly, virus titers in the supernatants of ZMPSTE24 knockdown cells as

149 quantified by $\mathrm{TCID}_{50}$ assay [9] showed a slight decrease relative to control

150 silenced cells and an average of 2.5-fold decrease was observed (Fig. 2C). To test

151 if siRNA induced silencing affected cell viability, MARC-145 cells were

152 transfected with either negative control siRNA or ZMPSTE24 siRNA (2

$153 \mathrm{pmoles} / \mu \mathrm{l}$ ) for $72 \mathrm{~h}$ and subjected to the cytotoxicity assay. There was no

154 significant difference $(\mathrm{p}>0.05)$ in cell viability between negative control siRNA

155 and ZMPSTE24 siRNA transfected groups (Fig. 2D). Overall, results showed that

156 silencing of endogenous ZMPSTE24 does not appear to have a significant impact

157 on PRRSV replication. This observation could be due to the knockdown

158 efficiency (74\%) achieved in our hands, while previous studies used cells derived

159 from gene knockout animals [5]. Additionally, it is possible that transient

160 silencing of ZMPSTE24 may not mimic the cells from gene knockout mice $[5,8]$. 
block entry of various enveloped viruses including IAV and arenaviruses [5, 16].

To examine whether overexpression of ZMPSTE24 restricts PRRSV entry, MARC-145 cells cultured in two 8-chamber slides were transfected with $0.3 \mu \mathrm{g}$ of each plasmid using the procedure as described earlier. PRRSV 23983 at an MOI of 4 was added to all wells. Two slides were fixed in $3.7 \%$ formaldehyde at either 3 or 24 hpi and then incubated with $0.2 \%$ Triton $x-100$. Then slides were incubated overnight with mouse monoclonal anti-Flag primary antibody (9A3) (Cell Signaling Technology, cat. no. 8146S) at 1:1600 dilution. The slides were

170 then incubated for $1 \mathrm{~h}$ with Alexa fluor 647 conjugated goat anti-mouse IgG $2^{\circ} \mathrm{Ab}$ 171 (Abcam) at 1:200 dilution and then with anti-PRRSV FITC-SDOW-17. Cells 172 were observed using Olympus Fluoview FV1200 Laser Scanning Confocal 173 Microscope at 40x magnification. There were no significant differences $(\mathrm{p}>0.05)$

174 in the number of PRRSV positive cells or total viral RNA copies between the 175 vector control and ZMPSTE24 over-expressing cells at 3 hpi (Fig. 3A and 3B). 176 qRT-PCR showed approximately 900-fold change in ZMPSTE24 mRNA 177 transcript in ZMPSTE24 overexpressing cells compared to control vector, 178 confirming the successful transfection and over-expressing of ZMPSTE24 (Fig.

$1793 \mathrm{C}$ ). Our results are in indirect contrast to an earlier study which showed a 180 dramatic lower number of IAV virus positive cells compared to the vector control 181 as early as 2 hpi and persisted until 8 hpi [5]. This difference could be due to 182 different virus entry mechanisms or distinctive replication efficiency of each virus 183 in the respective cell culture model systems. PRRSV completes one infectious cycle in about $8 \mathrm{~h}$ [18]. We observed 185 that PRRSV colocalized with the early endosome marker EEA1 at 3 hpi, but most 186 disappeared from early endosome at 6 hpi (data not shown). Very few PRRSV 
187 colocalize with LAMP-1, a marker of late endosome/lysosome, during the first 6 188 hpi. Our data is consistent with one previous study showing colocalization of 189 PRRSV with EEA1 but not with the late endosomal marker [17]. However, a 190 more recent study showed colocalization of PRRSV with late endosome marker 191 [18]. Therefore, the existing data remain controversial regarding whether PRRSV 192 enters cells through later endosomes. We observed extensive colocalization 193 between ZMPSTE24 and PRRSV at 3 hpi (Fig. 3D), suggesting virus entry into 194 early endosomes was not disturbed by ZMPSTE24 overexpression. However, 195 little or no colocalization between PRRSV and ZMPSTE24 was observed at 24 196 hpi (Fig. 3D), suggesting that restriction of PRRSV replication in cells with 197 ZMPSTE24 overexpression likely occurred after 3 hpi. It is known that IFITM3 198 and ZMPSTE24 work together to trap enveloped virus in the endolysosomal 199 compartment for degradation [5, 10]. Future studies on colocalization of PRRSV 200 with late endosome/lysosome marker (LAMP-1) in ZMPSTE24 overexpressing 201 cells may reveal more information on whether PRRSV is also being degraded in 202 late endosome/lysosome.

We observed a difference in the morphology of virus infected cells

204 between the cells not expressing ZMPSTE24 and the cells over-expressing 205 ZMPSTE24. Specifically, an increased number of cell surface protrusions was 206 observed in the vector control group at $3 \mathrm{hpi}$ (data not shown). One possible 207 explanation is that ZMPSTE24 overexpression may suppress the formation of cell 208 surface protrusions mediated by small GTPase such as Rho [6, 7, 13]. Future 209 investigation may clarify if ZMPSTE24 also interferes with PRRSV intercellular 210 spread. Overall, the data suggest that overexpression of ZMPSTE24 does not 211 significantly impact virus entry and may limit virus replication via a post-entry 212 step. Although ZMPSTE24 inhibited several RNA and DNA viruses requiring pH 
213 dependent fusion, it failed to inhibit murine leukemia virus [5]. Alternatively, 214 ZMPSTE24 may also bind to other substrates for its antiviral activity [10]. The 215 detailed mechanism by which overexpression of ZMPSTE24 restricts PRRSV 216 replication needs to be examined in future studies.

217 In conclusion, overexpression of ZMPSTE24 reduced PRRSV infection in 218 MARC-145 cells. There was no significant difference in the percentage of cells 219 positive for PRRSV and viral RNA copies in ZMPSTE24 overexpressing cells as 220 compared to the vector control cells at 3 hpi. This suggests that ZMPSTE24 may 221 inhibit PRRSV at a post-entry step. The role of endogenous ZMPSTE24 in 222 PRRSV replication seems to be limited in our experimental model system and 223 needs to be investigated further in future studies. 


\section{Author Contributions}

240 XW designed the study, analyzed the data, and wrote the manuscript. PK

241 conducted experiments, analyzed the results and wrote the manuscript. EN and SL

242 provided reagents/analytic tools. MH contributed to confocal microscopy and

243 analysis.

245 Compliance with Ethical Standard:

246 Funding: This study is partly supported by Animal Health and Production and

247 Animal Products: Animal Health and Disease (grant no. 2021-67016-

24834460 /project accession no. 1025717) from the USDA National Institute of Food

249 and Agriculture (NIFA) and Hatch (SD00H660-19) and Hatch Multi State

250 (SD00R656-16) from USDA NIFA and South Dakota Agricultural Experiment

251 Station.

252 Conflict of Interest: The authors declare no conflict of interest. 
1. Bailey CC, Zhong G, Huang IC, Farzan M (2014) IFITM-Family Proteins: The Cell's First Line of Antiviral Defense. Annu Rev Virol 1:261-283

2. Barrowman J, Michaelis S (2009) ZMPSTE24, an integral membrane zinc metalloprotease with a connection to progeroid disorders. Biological chemistry 390:761-773

3. Feeley EM, Sims JS, John SP, Chin CR, Pertel T, Chen LM, Gaiha GD, Ryan BJ, Donis RO, Elledge SJ, Brass AL (2011) IFITM3 inhibits influenza A virus infection by preventing cytosolic entry. PLoS pathogens 7:e1002337

4. Fong LG, Ng JK, Meta M, Coté N, Yang SH, Stewart CL, Sullivan T, Burghardt A, Majumdar S, Reue K, Bergo MO, Young SG (2004) Heterozygosity for Lmna deficiency eliminates the progeria-like phenotypes in Zmpste24-deficient mice. Proceedings of the National Academy of Sciences of the United States of America 101:18111-18116

5. Fu B, Wang L, Li S, Dorf ME (2017) ZMPSTE24 defends against influenza and other pathogenic viruses. The Journal of Experimental Medicine 214:919

6. Guo R, Katz BB, Tomich JM, Gallagher T, Fang Y (2016) Porcine Reproductive and Respiratory Syndrome Virus Utilizes Nanotubes for Intercellular Spread. Journal of virology 90:5163-5175

7. Kumar A, Kim JH, Ranjan P, Metcalfe MG, Cao W, Mishina M, Gangappa S, Guo Z, Boyden ES, Zaki S, York I, García-Sastre A, Shaw M, Sambhara S (2017) Influenza virus exploits tunneling nanotubes for cell-to-cell spread. Scientific Reports 7:40360

8. Lam JKW, Chow MYT, Zhang Y, Leung SWS (2015) siRNA Versus miRNA as Therapeutics for Gene Silencing. Molecular Therapy - Nucleic Acids 4:e252

9. Lei C, Yang J, Hu J, Sun X (2021) On the Calculation of TCID(50) for Quantitation of Virus Infectivity. Virol Sin 36:141-144

10. Li S, Fu B, Wang L, Dorf ME (2017) ZMPSTE24 Is Downstream Effector of Interferon-Induced Transmembrane Antiviral Activity. DNA Cell Biol 36:513-517

11. Livak KJ, Schmittgen TD (2001) Analysis of Relative Gene Expression Data Using Real-Time Quantitative PCR and the 2- $\Delta \Delta C T$ Method. Methods 25:402-408

12. Lunney JK, Fang Y, Ladinig A, Chen N, Li Y, Rowland B, Renukaradhya GJ (2016) Porcine Reproductive and Respiratory Syndrome Virus (PRRSV): Pathogenesis and Interaction with the Immune System. Annual review of animal biosciences 4:129-154

13. Panasiuk M, Rychłowski M, Derewońko N, Bieńkowska-Szewczyk K (2018) Tunneling Nanotubes as a Novel Route of Cell-to-Cell Spread of Herpesviruses. Journal of virology 92

14. Schneider WM, Chevillotte MD, Rice CM (2014) Interferon-stimulated genes: a complex web of host defenses. Annual review of immunology 32:513-545

15. Smith S, Weston S, Kellam P, Marsh M (2014) IFITM proteins-cellular inhibitors of viral entry. Current opinion in virology 4:71-77

16. Stott RJ, Foster TL (2021) Inhibition of arenavirus entry and replication by the cell-intrinsic restriction factor ZMPSTE24 is enhanced by IFITM antiviral activity. bioRxiv:2021.2004.2012.439453

17. Van Gorp H, Van Breedam W, Delputte PL, Nauwynck HJ (2009) The porcine reproductive and respiratory syndrome virus requires trafficking through CD163positive early endosomes, but not late endosomes, for productive infection. Archives of virology 154:1939-1943

18. Zhang A, Duan H, Zhao H, Liao H, Du Y, Li L, Jiang D, Wan B, Wu Y, Ji P, Zhou E-M, Zhang G (2020) Interferon-Induced Transmembrane Protein 3 Is a Virus- 

Syndrome Virus Replication by Blocking Viral Membrane Fusion. Journal of virology 94:e01350-01320

19. Zhang Q, Yoo D (2015) PRRS virus receptors and their role for pathogenesis. 
Fig. 1 Over-expression of ZMPSTE24 in MARC-145 cells reduces PRRSV replication. Standard deviation and mean of three replicates are shown for each graph. A: Western blot analysis showing the expression of ZMPSTE24 in cells transfected with ZMPSTE24 expressing plasmid. Lanes 1-3 were transfected with PRRSV infected cells at 24 hpi. * indicates $\mathrm{p}<0.05$. C: Immunofluorescence staining of virus-infected cells at $24 \mathrm{hpi}$. Top panel shows the bright field image and the lower panel shows PRRSV positive staining. D: Flow cytometry analysis of virus-infected cells at 24 hpi. * indicates $p<0.05$. E: CCK-8 assay was used to compare cell viability between vector control and ZMPSTE24 containing plasmid transfected cells. No significant difference $(p>0.05)$ was observed.

Fig. 2 Silencing of endogenous ZMPSTE24 slightly affects PRRSV replication.

359 Standard deviation and mean of three replicates are shown for each graph. A: qRT-PCR showed an average ZMPSTE24 knockdown efficiency of 74\%. B: The relative fold of change in viral RNA copies in ZMPSTE24 siRNA transfected cells compared to control silencing RNA transfected cells. No significant difference $(p>0.05)$ was observed between the two groups. C: TCID $_{50}$ assay showing virus titers in the supernatants of ZMPSTE24 silencing cells as compared to the control silencing cells. No significant difference between the two groups was observed $(p>0.05)$. D: Cell viability was not significantly $(p>0.05)$ affected 367 by the transient silencing of ZMPSTE24.

368 Fig. 3 Over-expression of ZMPSTE24 does not affect virus entry into MARC-145 369 cells. A: Quantitative analysis of PRRSV positive cells in ZMPSTE24 expressing 
370 cells and vector control cells at 3 hpi. Percentage of PRRSV positive cells was

371 determined by dividing the green (FITC-SDOW-17) staining cells by total number

372 of cells (DAPI stained, blue) in each microscopic field. The average and standard

373 deviations of five different microscopic fields are shown. The percentage of red

374 staining (Alexa 647, ZMPSTE24 expressing cells) that is positive for PRRSV was

375 determined similarly. Red and green co-staining cells were counted and divided

376 by the total number of red staining cells. No significant difference $(p>0.05)$ was

377 observed between vector control and ZMPSTE24 transfected group. B: The

378 relative fold change in viral RNA copies in ZMPSTE24 transfected cells when

379 compared to vector control at $3 \mathrm{hpi}$ as determined by qRT-PCR. Standard

380 deviation and mean of three replicates are shown. C: qRT-PCR showing the

381 relative fold increase in ZMPSTE24 mRNA level in ZMPSTE24 transfected cells

382 compared to vector control cells. Standard deviation and mean of three replicates

383 are shown. D: Colocalization of PRRSV N protein with exogenous ZMPSTE24.

384 Representative images show the extensive colocalization of PRRSV N with

385 ZMPSTE24 at 3 hpi and little or no colocalization of PRRSV N protein with

386 ZMPSTE24 at 24 hpi. Green: PRRSV N protein. Red: Flag-tagged ZMPSTE24.

387 Blue: DAPI nuclei. 
V V V Z Z Z

ACTIN

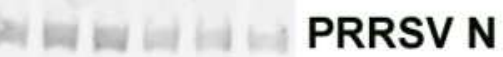

398

\section{ZMPSTE24-FLAG}

$399 \quad$ Fig. 1B

400

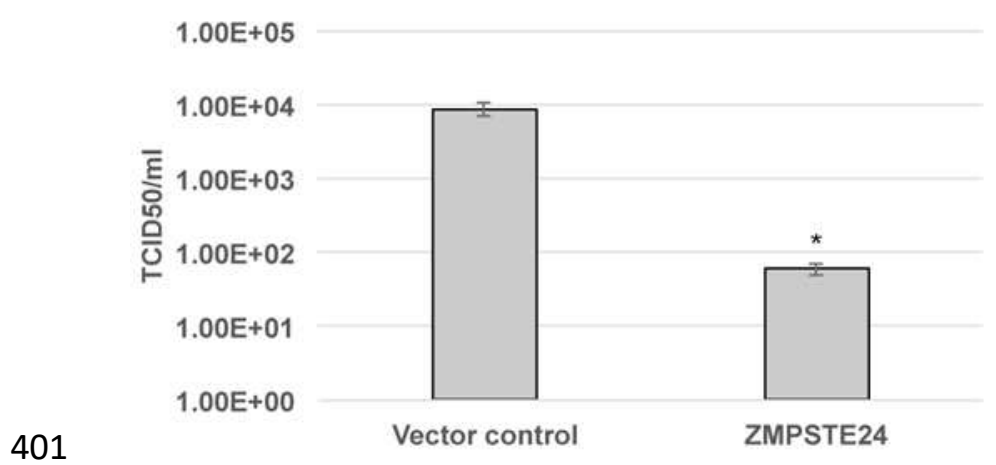

402 Fig. 1C

403

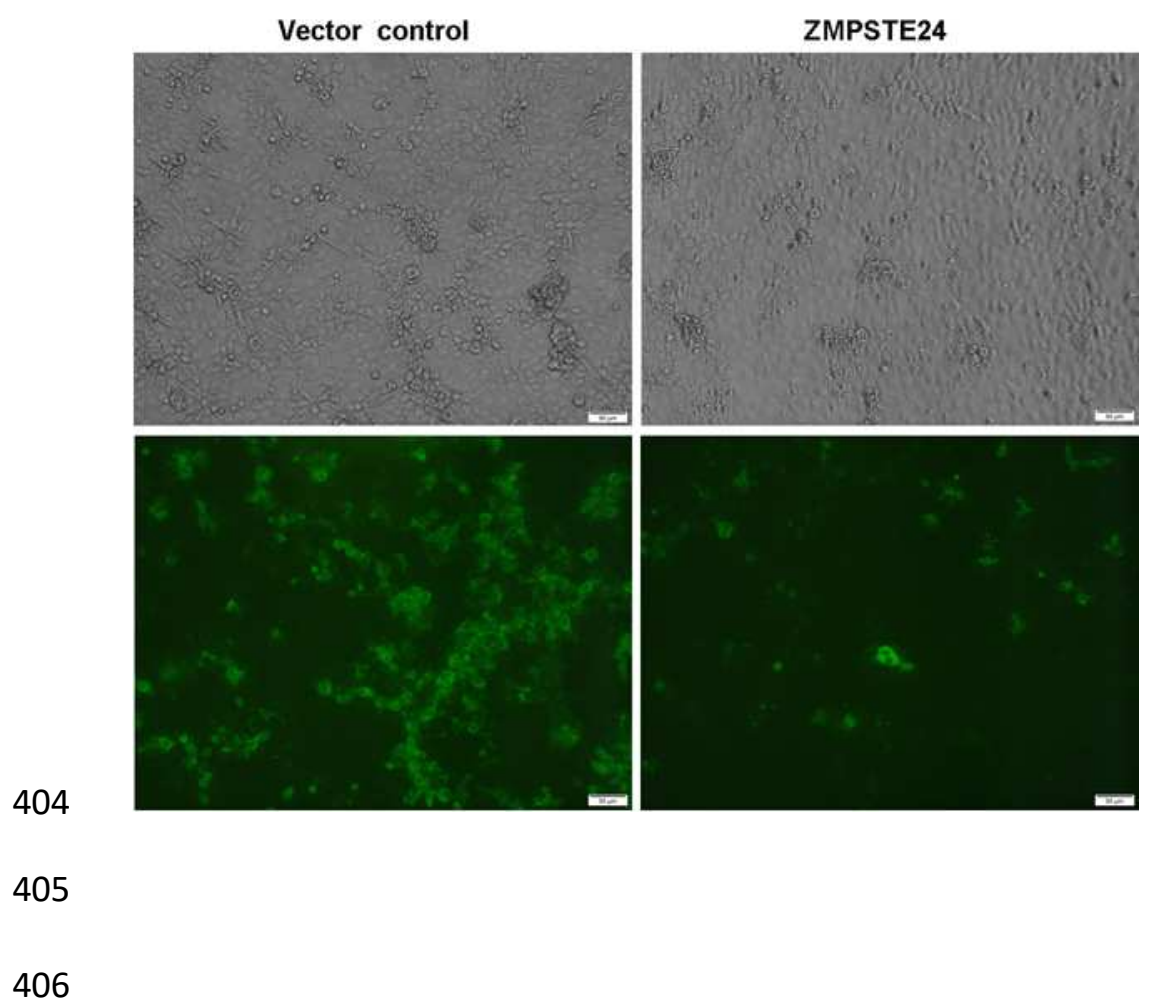


Fig. 1D

409

410

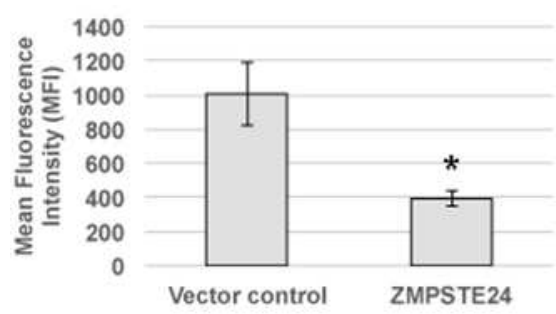

411

414 Fig. 1E

415

416

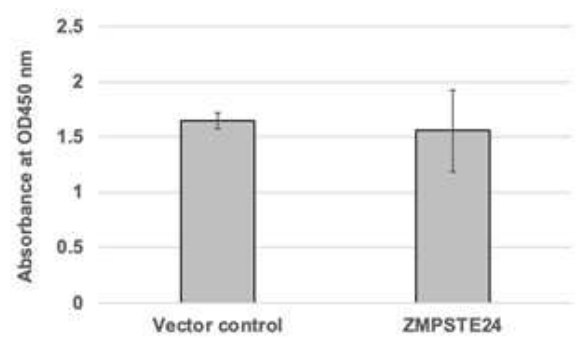

417

418

419

420

421

422

423

424

425

426 
Fig. 2A

428

429

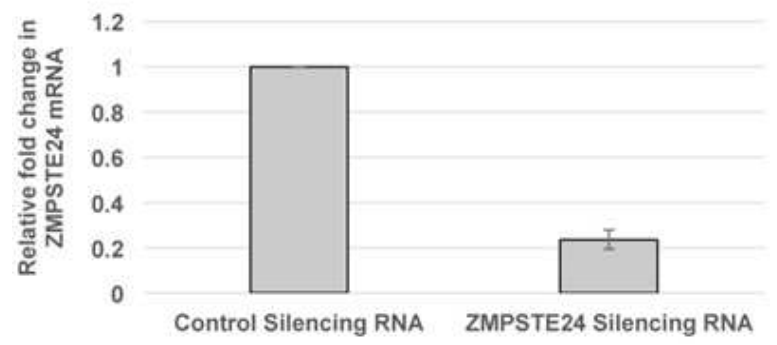

430

431

Fig. 2B

432

433

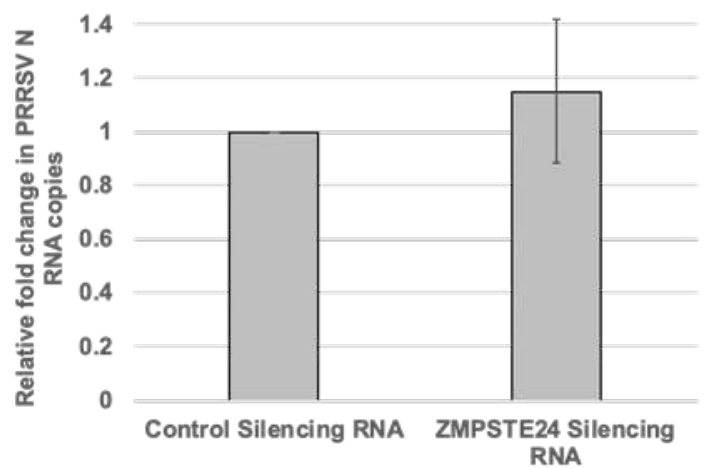

434

435 Fig. 2C

436

437

438

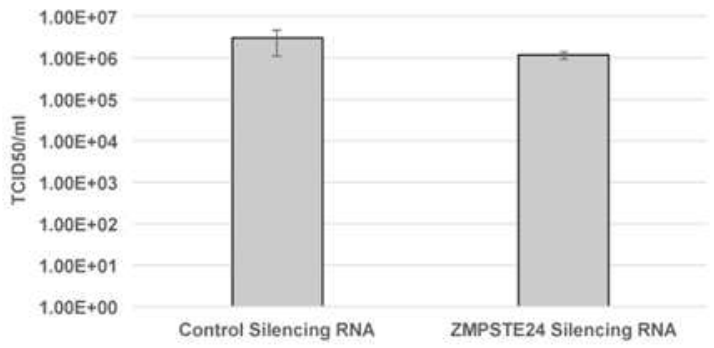

439

440

441 
Fig. 2D

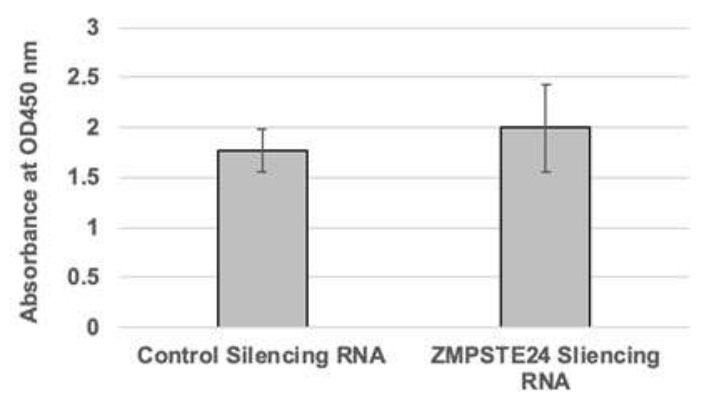

443

444

445

446

Fig. 3A

447

448

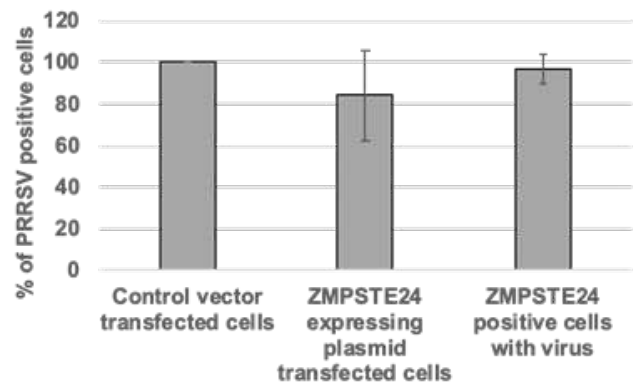

449

450

451

452 Fig. 3B

453

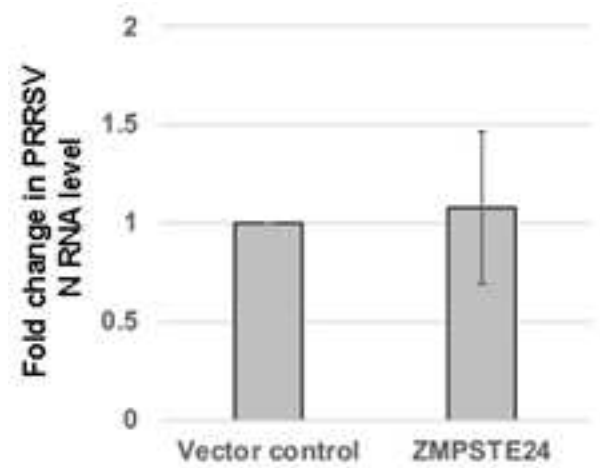

455 
456 Fig. 3C

457

458

459

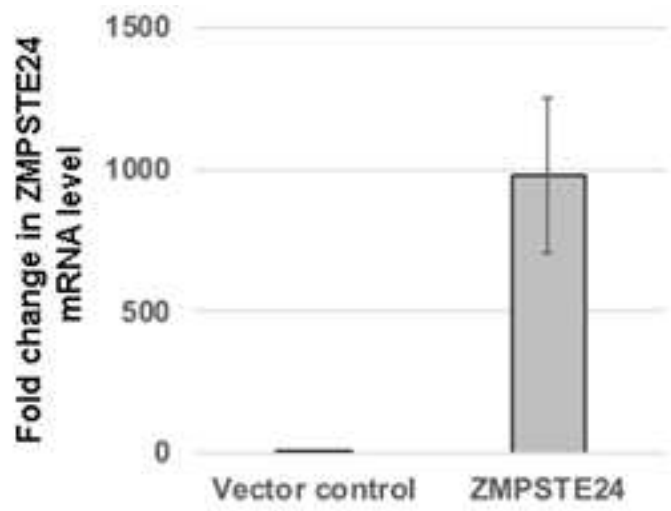

460

461

462

$463 \quad$ Fig. 3D

464

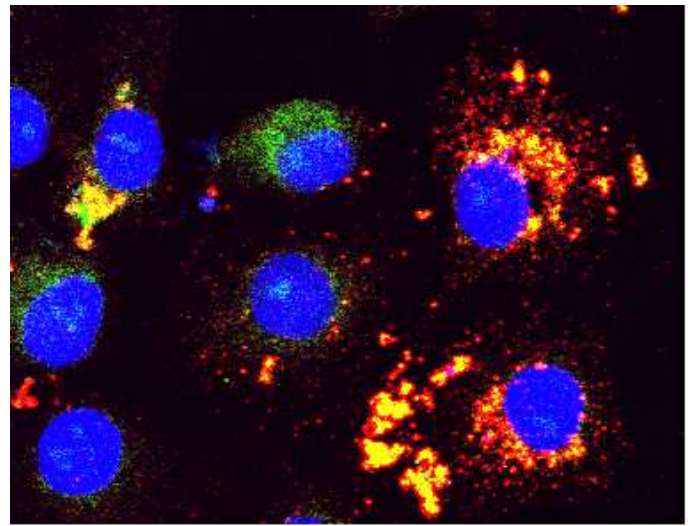

$3 \mathrm{hpi}$

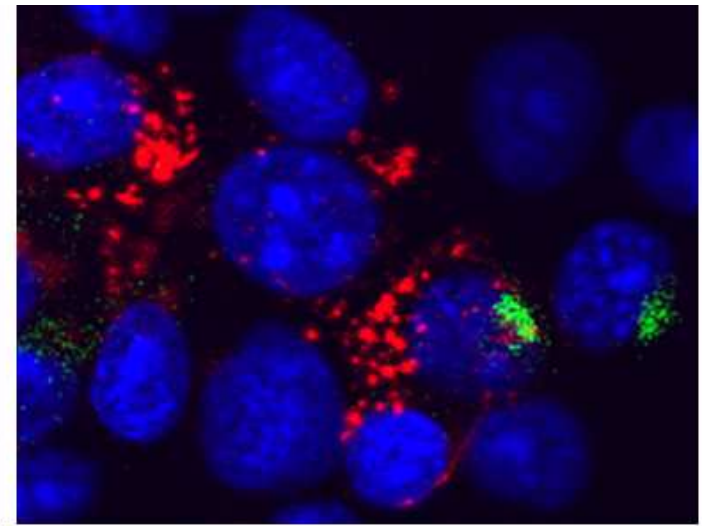

24 hpi

465 